\title{
A Sum Operator Method for the Existence and Uniqueness of Positive Solutions to a System of Nonlinear Fractional Integral Equations
}

\author{
Jing $W u^{1}$ and Tunhua $W u^{2}$ \\ ${ }^{1}$ School of Economic Mathematics, Southwestern University of Finance and Economics, Chengdu, Sichuan 610074, China \\ ${ }^{2}$ School of Information and Engineering, Wenzhou Medical College, 325035, Wenzhou, Zhejiang, China \\ Correspondence should be addressed to Jing Wu; xinanwujing@163.com
}

Received 29 April 2013; Revised 7 June 2013; Accepted 27 June 2013

Academic Editor: Yonghong Wu

Copyright (C) $2013 \mathrm{~J}$. Wu and T. Wu. This is an open access article distributed under the Creative Commons Attribution License, which permits unrestricted use, distribution, and reproduction in any medium, provided the original work is properly cited.

This paper is concerned with the existence and uniqueness of positive solutions for a Volterra nonlinear fractional system of integral equations. Our analysis relies on a fixed point theorem of a sum operator. The conditions for the existence and uniqueness of a positive solution to the system are established. Moreover, an iterative scheme is constructed for approximating the solution. The case of quadratic system of fractional integral equations is also considered.

\section{Introduction}

Fractional calculus has been used for the study of problems in various fields of sciences, such as Abel integral equation and viscoelasticity, analysis of feedback amplifiers, capacitor theory, fractances, generalized voltage dividers, and engineering and biological sciences. In [1], Kilbas et al. give a survey of research in fractional calculus and its applications in mathematical analysis such as ODEs, PDEs, convolution integral equations, and theory of generating equations. Particularly, fractional differential equations have successful applications in nonlinear oscillation analysis of earthquakes, seepage flow in porous media [2], and fluid dynamic models for traffic flow [3], as the fractional derivatives can eliminate the deficiency of continuum traffic flow.

Open problems in this field are finding easy and effective methods for solving the equations. In recent years, many techniques of functional analysis, such as the fixed point theory, the Banach contraction principle, and the LeraySchauder theory, are applied for solving the nonlinear fractional differential equations [4-11]. Iterative techniques [12$14]$ and the upper and lower solution method $[15,16]$ are also introduced to investigate the existence and uniqueness of the solutions to nonlinear fractional order differential equations with various boundary conditions.

Recently, prompted by the applications in physics, the following nonlinear quadratic system of integral equations and its generalizations have provoked some interest:

$$
\begin{array}{r}
1=\varphi_{i}(t)+\lambda_{i} \varphi_{i}(t) \int_{0}^{t} \frac{(t-s)^{\alpha_{i}-1}}{\Gamma\left(\alpha_{i}\right)} \varphi_{i}(s) d s \\
\alpha_{i} \in(0,1), i=1,2, \ldots, n .
\end{array}
$$

Salem [17] applied Krasnoselskii's fixed point theorem to obtain the existence of solutions for the system:

$$
\begin{array}{r}
x_{i}(t)=\varphi_{i}(t)+\lambda_{i} I^{\alpha_{i}}\left[f_{i}(x(t))+g_{i}(x(t))\right], \\
t \in[0,1], \alpha_{i} \in(0,1), 1 \leq i \leq n,
\end{array}
$$

under the assumptions that $f_{i}:[0, \infty)^{n} \rightarrow[0, \infty)$ is continuous nondecreasing for all variables, and $g_{i}:[0, \infty)^{n} \rightarrow$ $[0, \infty)$ is continuous nonincreasing for all variables, where $[0, \infty)^{n}$ denotes the $n$-products $[0, \infty) \times[0, \infty) \cdots \times[0, \infty)$ and $x=\left(x_{1}, x_{2}, \ldots, x_{n}\right)$. For the physical point of view, only 
positive solutions are interesting. A simple form of the system (2):

$$
x(t)=\int_{0}^{t} \frac{(t-s)^{\alpha-1}}{\Gamma(\alpha)} f(x(s)) d s
$$

has been studied in $[18,19]$.

The aim of this paper is to study the existence and uniqueness of positive solutions for the following Volterra nonlinear fractional system of integral equations:

$$
\begin{array}{r}
x_{i}(t)=\int_{0}^{t} \frac{(t-s)^{\alpha_{i}-1}}{\Gamma(\alpha)}\left[f_{i}(s, x(s))+g_{i}(s, x(s))\right] d s, \\
t \in[0,1], \alpha_{i} \in(0,1), 1 \leq i \leq n .
\end{array}
$$

Our main interest is to give some alternative answers to the main results of papers [17-19]. By using a fixed point theorem of a sum operator, we not only obtain the existence and uniqueness of positive solutions for the system (4), but also construct some sequences for approximating the unique solution.

\section{Basic Definitions and Preliminaries}

For the convenience of the reader, we present here some definitions, lemmas, and basic results that will be used in the proofs of our main results.

Definition 1 (see [1]). The fractional integral of order $\alpha>0$ of a function $f:(0,+\infty) \rightarrow R$ is given by

$$
I_{0+}^{\alpha} f(t)=\frac{1}{\Gamma(\alpha)} \int_{0}^{t}(t-s)^{\alpha-1} f(s) d s,
$$

provided that the right-hand side is defined pointwisely on $(0,+\infty)$, and $\Gamma(\alpha)$ denotes the gamma function.

Suppose that $E$ is a real Banach space which is partially ordered by a cone $P \subset E$; that is, $x \leq y$ if and only if $y-x \in P$. If $x \leq y$ and $x \neq y$, then we denote $x<y$ or $y>x$. By $\theta$ we denote the zero element of $E$. Recall that a nonempty closed convex set $P \subset E$ is a cone if it satisfies (i) $x \in P$, $\lambda \geq 0 \Rightarrow \lambda x \in P$; (ii) $x \in P,-x \in P \Rightarrow x=\theta$.

Let $P^{\circ}=\{x \in P \mid x$ is an interior point of $P\}$, and then a cone $P$ is said to be solid if $P^{\circ}$ is nonempty. Moreover, $P$ is called normal if there exists a constant $N>0$ such that, for all $x, y \in E, \theta \leq x \leq y$ implies $\|x\| \leq N\|y\|$; in this case $N$ is called the normality constant of $P$. If $x_{1}, x_{2} \in E$, the set $\left[x_{1}, x_{2}\right]=\left\{x \in E \mid x_{1} \leq x \leq x_{2}\right\}$ is called the order interval between $x_{1}$ and $x_{2}$. We say that an operator $A: E \rightarrow E$ is increasing (decreasing) if $x \leq y$ implies $A x \leq A y(A x \geq A y)$. For all $x, y \in E$, the notation $x \sim y$ means that there exist $\lambda>0$ and $\mu>0$ such that $\lambda x \leq y \leq \mu x$. Clearly, $\sim$ is an equivalence relation. Given $h>\theta$ (i.e., $h \geq \theta$ and $h \neq \theta$ ), we denote by $P_{h}$ the set $P_{h}=\{x \in E \mid x \sim h\}$. It is easy to see that $P_{h} \subset P$.

Definition 2. Let $D=P$ or $D=P^{\circ}$ and $\gamma$ be a real number with $0 \leq \gamma<1$. An operator $A: P \rightarrow P$ is said to be $\gamma$ concave if it satisfies

$$
A(t x) \geq t^{\gamma} A x, \quad \forall t \in(0,1), x \in D .
$$

Definition 3. An operator $A: E \rightarrow E$ is said to be homogeneous if it satisfies

$$
A(t x)=t A x, \quad \forall t>0, x \in E .
$$

An operator $A: P \rightarrow P$ is said to be subhomogeneous if it satisfies

$$
A(t x) \geq t A x, \quad \forall t \in(0,1), x \in P .
$$

In the recent paper [20], Zhai and Anderson considered the following sum operator equation:

$$
A x+B x+C x=x
$$

where $A$ is an increasing $\gamma$-concave operator, $B$ is an increasing subhomogeneous operator, and $C$ is a homogeneous operator. They established the existence and uniqueness of positive solutions for the above equation, and when $C$ is a null operator, they present the following interesting result.

Lemma 4 (see [20]). Let $P$ be a normal cone in a real Banach space $E$, let $A: P \rightarrow P$ be an increasing $\gamma$-concave operator, and let $B: P \rightarrow P$ be an increasing subhomogeneous operator. Assume that

(1) there is $h>\theta$ such that $A h \in P_{h}, B h \in P_{h}$;

(2) there exists a constant $\delta>0$ such that $A x \geq \delta B x$, for all $x \in P$.

Then the operator equation $A x+B x=x$, has a unique solution $x^{*}$ in $P_{h}$. Moreover, constructing successively the sequence $y_{n}=A y_{n-1}+B y_{n-1}, n=1,2, \ldots$ for any initial value $y_{0} \in P_{h}$, we have $y_{n} \rightarrow x^{*}$, as $n \rightarrow \infty$.

\section{Main Results}

In this section, we apply Lemma 4 to study problem (4), and we obtain some new results on the existence and uniqueness of positive solutions.

Now by $C[0,1]$, we mean the Banach space of continuous functions on $[0,1]$ with the usual max-norm $\|\cdot\|$. Also, recall the Banach space of the cartesian product $E=$ : $C[0,1] \times C[0,1] \times \cdots \times C[0,1]$ equipped by the norm $\|x\|=$ : $\max _{1 \leq i \leq n}\left\|x_{i}\right\|$. Notice that this space can be equipped with a partial order:

$$
\begin{array}{r}
x, y \in E, x \leq y \Longleftrightarrow x_{i}(t) \leq y_{i}(t), \\
t \in[0,1], i=1,2, \ldots, n .
\end{array}
$$

Set $P=\{x \in E \mid x(t) \geq 0, t \in[0,1]\}$, the standard cone. It is clear that $P$ is a normal cone in $E$ and the normality constant is 1. Take $h(t)=\left(h_{1}(t), h_{2}(t), \ldots, h_{n}(t)\right)$ and $h_{i}(t)=t^{\alpha_{i}}$,

$$
P_{h}=\{x \in P \mid x \sim h\} .
$$

Theorem 5. Assume that

(S1) for all $i, f_{i}, g_{i}:[0,1] \times[0, \infty)^{n} \rightarrow[0, \infty)$ are continuous and increasing with respect to the arguments $x_{i}$, and $g_{i}(t, 0,0, \ldots, 0)>0$ for any $t \in[0,1]$; 
(S2) for all $i, g_{i}\left(t, x_{1}, x_{2}, \ldots, \tau x_{i}, \ldots, x_{n}\right) \geq \tau g_{i}\left(t, x_{1}, x_{2}\right.$, $\left.\ldots, x_{i}, \ldots, x_{n}\right)$ for $\tau \in(0,1), t \in[0,1], x_{i} \in[0,+\infty)$ and there exist constants $\gamma_{i} \in(0,1)$ such that

$$
f_{i}\left(t, x_{1}, x_{2}, \ldots, \tau x_{i}, \ldots, x_{n}\right) \geq \tau^{\gamma} f_{i}\left(t, x_{1}, x_{2}, \ldots, x_{i}, \ldots, x_{n}\right)
$$

for $\tau \in(0,1), t \in[0,1], x_{i} \in[0,+\infty), i=1,2, \ldots, n ;$

(S3) there exists $\delta_{i}>0$ such that

$$
\begin{array}{r}
f_{i}\left(t, x_{1}, x_{2}, \ldots, x_{i}, \ldots, x_{n}\right) \geq \delta_{i} g_{i}\left(t, x_{1}, x_{2}, \ldots, x_{i}, \ldots, x_{n}\right), \\
t \in[0,1], x_{i} \geq 0, i=1,2, \ldots, n .
\end{array}
$$

Then problem (4) has a unique positive solution $x^{*}$ in $P_{h}$. Moreover, for any initial value $x^{(0)}=\left(x_{1}^{(0)}, x_{2}^{(0)}, \ldots, x_{n}^{(0)}\right) \in P_{h}$, constructing successively the sequence

$$
\begin{aligned}
& x_{i}^{(m+1)}(t) \\
& =\int_{0}^{t} \frac{(t-s)^{\alpha_{i}-1}}{\Gamma\left(\alpha_{i}\right)} \\
& \quad \times\left[f_{i}\left(s, x_{1}^{(m)}(s), x_{2}^{(m)}(s), \ldots, x_{i}^{(m)}(s), \ldots, x_{n}^{(m)}(s)\right)\right. \\
& \quad+g_{i}\left(s, x_{1}^{(m)}(s), x_{2}^{(m)}(s), \ldots,\right. \\
& \left.\left.\quad x_{i}^{(m)}(s), \ldots, x_{n}^{(m)}(s)\right)\right] d s, \\
& m=0,1, \ldots,
\end{aligned}
$$

then $x^{(m)} \rightarrow x^{*}$ as $m \rightarrow \infty$.

Proof. To begin with, we define the following operators $A, B$ : $P \rightarrow E$ by

$$
\begin{gathered}
A x=\left(A_{1} x_{1}, A_{2} x_{2}, \ldots, A_{n} x_{n}\right), \\
B x=\left(B_{1} x_{1}, B_{2} x_{2}, \ldots, B_{n} x_{n}\right),
\end{gathered}
$$

where

$$
\begin{aligned}
& A_{i} x_{i}=\int_{0}^{t} \frac{(t-s)^{\alpha_{i}-1}}{\Gamma\left(\alpha_{i}\right)} f_{i}(s, x(s)) d s, \\
& B_{i} x_{i}=\int_{0}^{t} \frac{(t-s)^{\alpha_{i}-1}}{\Gamma\left(\alpha_{i}\right)} g_{i}(s, x(s)) d s .
\end{aligned}
$$

Thus $x$ is the positive solution of problem (4) if and only if $x=A x+B x$. From $(S 0)$ and (S1), we know that $A: P \rightarrow$ $P, B: P \rightarrow P$. In the sequel we check that $A, B$ satisfy all assumptions of Lemma 4.
Firstly, we prove that $A, B$ are two increasing operators. In fact, by (S0) and (S1), for $x, y \in P$ with $x \geq y$, we know that $x_{i}(t) \geq y_{i}(t), t \in[0,1], i=1,2, \ldots, n$, and obtain

$$
\begin{aligned}
A_{i} x_{i}= & \int_{0}^{t} \frac{(t-s)^{\alpha_{i}-1}}{\Gamma\left(\alpha_{i}\right)} \\
& \quad \times f_{i}\left(s, x_{1}(s), x_{2}(s), \ldots, x_{i}(s), \ldots, x_{n}(s)\right) d s \\
\geq & \int_{0}^{t} \frac{(t-s)^{\alpha_{i}-1}}{\Gamma\left(\alpha_{i}\right)} \\
& \times f_{i}\left(s, y_{1}(s), y_{2}(s), \ldots, y_{i}(s), \ldots, y_{n}(s)\right) d s \\
= & A_{i} y_{i} ;
\end{aligned}
$$

that is, $A x \geq A y$. Similarly, $B x \geq B y$.

Next we show that $A$ is a $\gamma$-concave operator and $B$ is a subhomogeneous operator. In fact, for any $\tau \in(0,1)$ and $x \in$ $P$, by $(S 1)$, we obtain

$$
\begin{aligned}
& A_{i}\left(\tau x_{i}\right)(t) \\
& =\int_{0}^{t} \frac{(t-s)^{\alpha_{i}-1}}{\Gamma\left(\alpha_{i}\right)} \\
& \quad \quad \times f_{i}\left(s, x_{1}(s), x_{2}(s), \ldots, \tau x_{i}(s), \ldots, x_{n}(s)\right) d s \\
& \geq \tau^{\gamma_{i}} \int_{0}^{t} \frac{(t-s)^{\alpha_{i}-1}}{\Gamma\left(\alpha_{i}\right)} \\
& \quad \times f_{i}\left(s, x_{1}(s), x_{2}(s), \ldots, x_{i}(s), \ldots, x_{n}(s)\right) d s \\
& =\tau^{\gamma_{i}} A_{i} x_{i}(t) .
\end{aligned}
$$

Consequently, $A(\tau x)(t) \geq \tau^{\gamma} A x$, where $\gamma=\max _{1 \leq i \leq n} \gamma_{i}$. Also, for any $\tau \in(0,1)$ and $x \in P$, by $(S 0)$ and $(S 1)$, we obtain

$$
\begin{aligned}
B_{i}\left(\tau x_{i}\right)(t) & \\
=\int_{0}^{t} \frac{(t-s)^{\alpha_{i}-1}}{\Gamma\left(\alpha_{i}\right)} & \quad \times g_{i}\left(s, x_{1}(s), x_{2}(s), \ldots, \tau x_{i}(s), \ldots, x_{n}(s)\right) d s \\
\geq \tau \int_{0}^{t} \frac{(t-s)^{\alpha_{i}-1}}{\Gamma\left(\alpha_{i}\right)} & \quad \times g_{i}\left(s, x_{1}(s), x_{2}(s), \ldots, x_{i}(s), \ldots, x_{n}(s)\right) d s \\
= & \tau B_{i} x_{i}(t) ;
\end{aligned}
$$

that is, $B(\tau x) \geq \tau B x$ for $\tau \in(0,1), x \in P$. So the operator $B$ is a subhomogeneous operator.

Now we show that $A h \in P_{h}, B h \in P_{h}$. In fact, by (S3), we have

$$
f_{i}(s, 1,1, \ldots, 1) \geq f_{i}(s, 0,0, \ldots, 0) \geq \delta_{i} g_{i}(s, 0,0, \ldots, 0)>0,
$$


and thus take

$$
M_{i}=\max _{0 \leq s \leq 1} f_{i}(s, 1,1, \ldots, 1), \quad m_{i}=\min _{0 \leq s \leq 1} f_{i}(s, 0,0, \ldots, 0) ;
$$

then $M_{i}, m_{i}>0$. Let

$$
\lambda=\min _{1 \leq i \leq n}\left\{\frac{m_{i}}{\alpha_{i} \Gamma\left(\alpha_{i}\right)}\right\}, \quad \mu=\max _{1 \leq i \leq n}\left\{\frac{M_{i}}{\alpha_{i} \Gamma\left(\alpha_{i}\right)}\right\} .
$$

It follows from $(S 1)$ that

$$
\begin{aligned}
A_{i} h_{i}(t) & =\int_{0}^{t} \frac{(t-s)^{\alpha_{i}-1}}{\Gamma\left(\alpha_{i}\right)} f_{i}\left(s, s^{\alpha_{1}}, s^{\alpha_{2}}, \ldots, s^{\alpha_{i}}, \ldots, s^{\alpha_{n}}\right) d s \\
& \geq \int_{0}^{t} \frac{(t-s)^{\alpha_{i}-1}}{\Gamma\left(\alpha_{i}\right)} f_{i}(s, 0,0, \ldots, 0) d s \\
& \geq m_{i} \int_{0}^{t} \frac{(t-s)^{\alpha_{i}-1}}{\Gamma\left(\alpha_{i}\right)} d s \\
& =\frac{m_{i}}{\alpha_{i} \Gamma\left(\alpha_{i}\right)} t^{\alpha_{i}} \geq \lambda t^{\alpha_{i}}=\lambda h_{i}(t), \\
A_{i} h_{i}(t) & =\int_{0}^{t} \frac{(t-s)^{\alpha_{i}-1}}{\Gamma\left(\alpha_{i}\right)} f_{i}\left(s, s^{\alpha_{1}}, s^{\alpha_{2}}, \ldots, s^{\alpha_{i}}, \ldots, s^{\alpha_{n}}\right) d s \\
& \leq \int_{0}^{t} \frac{(t-s)^{\alpha_{i}-1}}{\Gamma\left(\alpha_{i}\right)} f_{i}(s, 1,1, \ldots, 1) d s \\
& \leq M_{i} \int_{0}^{t} \frac{(t-s)^{\alpha_{i}-1}}{\Gamma\left(\alpha_{i}\right)} d s \\
& =\frac{M_{i}}{\alpha_{i} \Gamma\left(\alpha_{i}\right)} t^{\alpha_{i}} \leq \mu t^{\alpha_{i}}=\mu h_{i}(t) .
\end{aligned}
$$

So $\lambda h_{i}(t) \leq A_{i} h_{i}(t) \leq \mu h_{i}(t)$, and then $\lambda h(t) \leq A h(t) \leq \mu h(t)$, hence $A h \in P_{h}$. Similarly, from $g_{i}(s, 0,0, \ldots, 0)>0$ and (S1)-(S3), we easily prove $B h \in P_{h}$. Hence the condition (1) of Lemma 4 is satisfied.

In the following we show that the condition (2) of Lemma 4 is satisfied. For $x \in P$, from (S3), we have

$$
\begin{aligned}
& A_{i} x_{i}(t) \\
& =\int_{0}^{t} \frac{(t-s)^{\alpha_{i}-1}}{\Gamma\left(\alpha_{i}\right)} \\
& \times f_{i}\left(s, x_{1}(s), x_{2}(s), \ldots, x_{i}(s), \ldots, x_{n}(s)\right) d s \\
& \geq \delta_{i} \int_{0}^{t} \frac{(t-s)^{\alpha_{i}-1}}{\Gamma\left(\alpha_{i}\right)} \\
& \times g_{i}\left(s, x_{1}(s), x_{2}(s), \ldots, x_{i}(s), \ldots, x_{n}(s)\right) d s \\
& =\delta_{i} B_{i} x_{i}(t) \text {. }
\end{aligned}
$$

Take

$$
\delta=\min _{1 \leq i \leq n} \delta_{i}
$$

and then we have $A x \geq \delta B x, x \in P$. By Lemma 4, the operator equation $A x+B x=x$ has a unique solution $x^{*} \in P_{h}$; of course, $x^{*}$ is also a unique solution of problem (4). In addition, by $(S 1)$ we know that the unique solution is also positive.

Now for any initial value $x^{(0)}=\left(x_{1}^{(0)}, x_{2}^{(0)}, \ldots, x_{n}^{(0)}\right) \in P_{h}$, let us construct successively the sequence

$$
x_{i}^{(m)}=A_{i} x_{i}^{(m-1)}+B_{i} x_{i}^{(m-1)}, \quad m=1,2, \ldots,
$$

and we have $x_{i}^{(m)} \rightarrow x_{i}^{*}$ as $m \rightarrow \infty$, and then problem (4) has a unique positive solution $x^{(m)} \rightarrow x^{*}$ in $P_{h}$; that is, for any initial value $x^{(0)}=\left(x_{1}^{(0)}, x_{2}^{(0)}, \ldots, x_{n}^{(0)}\right) \in P_{h}$, constructing successively the sequence:

$$
\begin{aligned}
& x_{i}^{(m+1)}(t) \\
& =\int_{0}^{t} \frac{(t-s)^{\alpha_{i}-1}}{\Gamma\left(\alpha_{i}\right)} \\
& \quad \times\left[f_{i}\left(s, x_{1}^{(m)}(s), x_{2}^{(m)}(s), \ldots, x_{i}^{(m)}(s), \ldots, x_{n}^{(m)}(s)\right)\right. \\
& \quad+g_{i}\left(s, x_{1}^{(m)}(s), x_{2}^{(m)}(s), \ldots,\right. \\
& \left.\left.\quad x_{i}^{(m)}(s), \ldots, x_{n}^{(m)}(s)\right)\right] d s, \\
& m=0,1, \ldots,
\end{aligned}
$$

then $x^{(m)} \rightarrow x^{*}$ as $m \rightarrow \infty$.

Corollary 6. Assume that

(A1) for all $i, f_{i}:[0,1] \times[0, \infty)^{n} \rightarrow[0, \infty)$ is continuous and increasing with respect to the arguments $x_{i}$, and $f_{i}(t, 0,0, \ldots, 0)>0$ for any $t \in[0,1]$;

(A2) for all $i, i=1,2, \ldots, n$, there exists constant $\gamma_{i} \in(0,1)$ such that

$f_{i}\left(t, x_{1}, x_{2}, \ldots, \tau x_{i}, \ldots, x_{n}\right) \geq \tau^{\gamma} f_{i}\left(t, x_{1}, x_{2}, \ldots, x_{i}, \ldots, x_{n}\right)$

$$
\text { for } \tau \in(0,1), t \in[0,1], x_{i} \in[0,+\infty) \text {. }
$$

Then the problem

$$
\begin{array}{r}
x_{i}(t)=\int_{0}^{t} \frac{(t-s)^{\alpha_{i}-1}}{\Gamma(\alpha)} f_{i}(s, x(s)) d s, \\
t \in[0,1], \alpha_{i} \in(0,1), 1 \leq i \leq n,
\end{array}
$$


has a unique positive solution $x^{*}$ in $P_{h}$. Moreover, for any initial value $x^{(0)}=\left(x_{1}^{(0)}, x_{2}^{(0)}, \ldots, x_{n}^{(0)}\right) \in P_{h}$, constructing successively the sequence

$$
\begin{aligned}
& x_{i}^{(m+1)}(t) \\
& =\int_{0}^{t} \frac{(t-s)^{\alpha_{i}-1}}{\Gamma\left(\alpha_{i}\right)} \\
& \quad \times f_{i}\left(s, x_{1}^{(m)}(s), x_{2}^{(m)}(s), \ldots, x_{i}^{(m)}(s), \ldots, x_{n}^{(m)}(s)\right) d s, \\
& \quad m=0,1, \ldots,
\end{aligned}
$$

then $x^{(m)} \rightarrow x^{*}$ as $m \rightarrow \infty$.

In what follows, we establish the existence and uniqueness of positive solutions for the following system of quadratic integral equations of the fractional type:

$$
\begin{aligned}
& \varphi_{i}(t)=\int_{0}^{t} \frac{(t-s)^{\alpha_{i}-1}}{\Gamma(\alpha)} \varphi_{i}^{2}(s) d s+1, \\
& t \in[0,1], \alpha_{i} \in(0,1), 1 \leq i \leq n .
\end{aligned}
$$

Corollary 7. The system (31) has a unique positive solution.

Proof. Let $f_{i}\left(x_{1}, x_{2}, \ldots, x_{n}\right)=\left(x_{i}+1\right)^{2}$, and then $f_{i}$ satisfies $(A 1)$ and $(A 2)$ of Corollary 6 . Thus let $x=\left(x_{1}, x_{2}, \ldots, x_{n}\right)$ be the unique positive solution of (29), and then we have

$$
x_{i}(t)=\int_{0}^{t} \frac{(t-s)^{\alpha_{i}-1}}{\Gamma(\alpha)}\left(x_{i}(s)+1\right)^{2} d s ;
$$

that is

$$
x_{i}(t)+1=\int_{0}^{t} \frac{(t-s)^{\alpha_{i}-1}}{\Gamma(\alpha)}\left(x_{i}(s)+1\right)^{2} d s+1 .
$$

Let $\varphi_{i}=x_{i}+1$, and the $\varphi=\left(\varphi_{1}, \varphi_{2}, \ldots, \varphi_{n}\right)$ is a unique positive solution of (31).

\section{Acknowledgments}

This work is supported by the Natural Science Foundation of China (no. 21207103), Natural Science Foundation of Zhejiang Province (no. LY13H180012), Public Benefit Project of Zhejiang Province (no. 2012C31025), Scientific Research Project of Zhejiang Education Department (no. Y201222932), and Scientific Research Project of Wenzhou (no. G20110004).

\section{References}

[1] A. A. Kilbas, H. M. Srivastava, and J. J. Trujillo, Theory and Applications of Fractional Differential Equations, vol. 204, Elsevier Science B.V., Amsterdam, The Netherlands, 2006.

[2] Y. Cheng and G. Guozhu, "On the solution of nonlinear fractional order differential equation," Nonlinear Analysis. Theory, Methods \& Applications, vol. 63, pp. 971-976, 2005.
[3] I. Podlubny, Fractional Differential Equations, vol. 198 of Mathematics in Science and Engineering, Academic Press, San Diego, Calif, USA, 1999.

[4] M. Rehman and R. A. Khan, "Existence and uniqueness of solutions for multi-point boundary value problems for fractional differential equations," Applied Mathematics Letters, vol. 23, no. 9, pp. 1038-1044, 2010.

[5] X. Zhang, L. Liu, and Y. Wu, "Multiple positive solutions of a singular fractional differential equation with negatively perturbed term," Mathematical and Computer Modelling, vol. 55, no. 3-4, pp. 1263-1274, 2012.

[6] H. A. H. Salem, "On the fractional order $m$-point boundary value problem in reflexive Banach spaces and weak topologies," Journal of Computational and Applied Mathematics, vol. 224, no. 2, pp. 565-572, 2009.

[7] W. Jiang, "The existence of solutions to boundary value problems of fractional differential equations at resonance," Nonlinear Analysis. Theory, Methods \& Applications, vol. 74, no. 5, pp. 1987-1994, 2011.

[8] X. Zhang, L. Liu, and Y. Wu, "Existence results for multiple positive solutions of nonlinear higher order perturbed fractional differential equations with derivatives," Applied Mathematics and Computation, vol. 219, no. 4, pp. 1420-1433, 2012.

[9] C. S. Goodrich, "Positive solutions to boundary value problems with nonlinear boundary conditions," Nonlinear Analysis. Theory, Methods \& Applications, vol. 75, no. 1, pp. 417-432, 2012.

[10] B. Ahmad and J. J. Nieto, "Existence results for higher order fractional differential inclusions with nonlocal boundary conditions," Nonlinear Studies, vol. 17, no. 2, pp. 131-138, 2010.

[11] C. S. Goodrich, "Existence and uniqueness of solutions to a fractional difference equation with nonlocal conditions," Computers \& Mathematics with Applications, vol. 61, no. 2, pp. 191-202, 2011.

[12] X. Zhang, L. Liu, and Y. Wu, "The uniqueness of positive solution for a singular fractional differential system involving derivatives," Communications in Nonlinear Science and Numerical Simulation, vol. 18, pp. 1400-1409, 2013.

[13] X. Zhang and Y. Han, "Existence and uniqueness of positive solutions for higher order nonlocal fractional differential equations," Applied Mathematics Letters, vol. 25, no. 3, pp. 555-560, 2012.

[14] X. Zhang, L. Liu, Y. Wu, and Y. Lu, "The iterative solutions of nonlinear fractional differential equations," Applied Mathematics and Computation, vol. 219, no. 9, pp. 4680-4691, 2013.

[15] X. Zhang, L. Liu, B. Wiwatanapataphee, and Y. Wu, "Positive solutions of eigenvalue problems for a class of fractional differential equations with derivatives," Abstract and Applied Analysis, vol. 2012, Article ID 512127, 16 pages, 2012.

[16] X. Zhang, L. Liu, and Y. Wu, "The eigenvalue problem for a singular higher order fractional differential equation involving fractional derivatives," Applied Mathematics and Computation, vol. 218, no. 17, pp. 8526-8536, 2012.

[17] H. A. H. Salem, "On the existence of continuous solutions for a singular system of non-linear fractional differential equations," Applied Mathematics and Computation, vol. 198, no. 1, pp. 445452, 2008.

[18] W. Mydlarczyk, "The existence of nontrivial solutions of Volterra equations," Mathematica Scandinavica, vol. 68, no. 1, pp. 83-88, 1991. 
[19] W. Mydlarczyk, "A nonlinear Abel equation on the whole line," Nonlinear Analysis. Theory, Methods \& Applications, vol. 45, no. 3, pp. 273-279, 2001.

[20] C. Zhai and D. R. Anderson, "A sum operator equation and applications to nonlinear elastic beam equations and LaneEmden-Fowler equations," Journal of Mathematical Analysis and Applications, vol. 375, no. 2, pp. 388-400, 2011. 


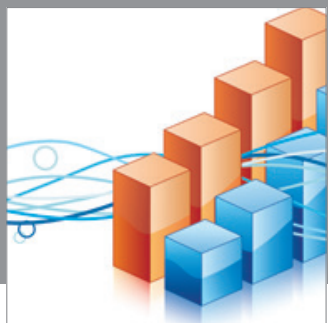

Advances in

Operations Research

mansans

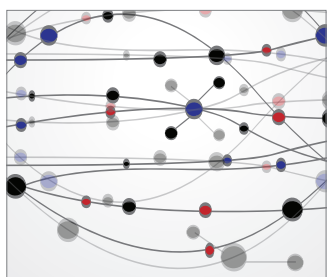

The Scientific World Journal
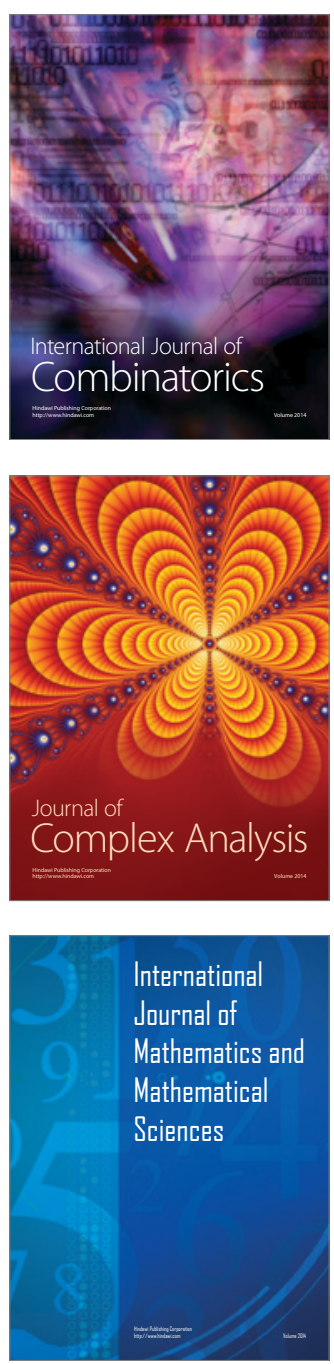
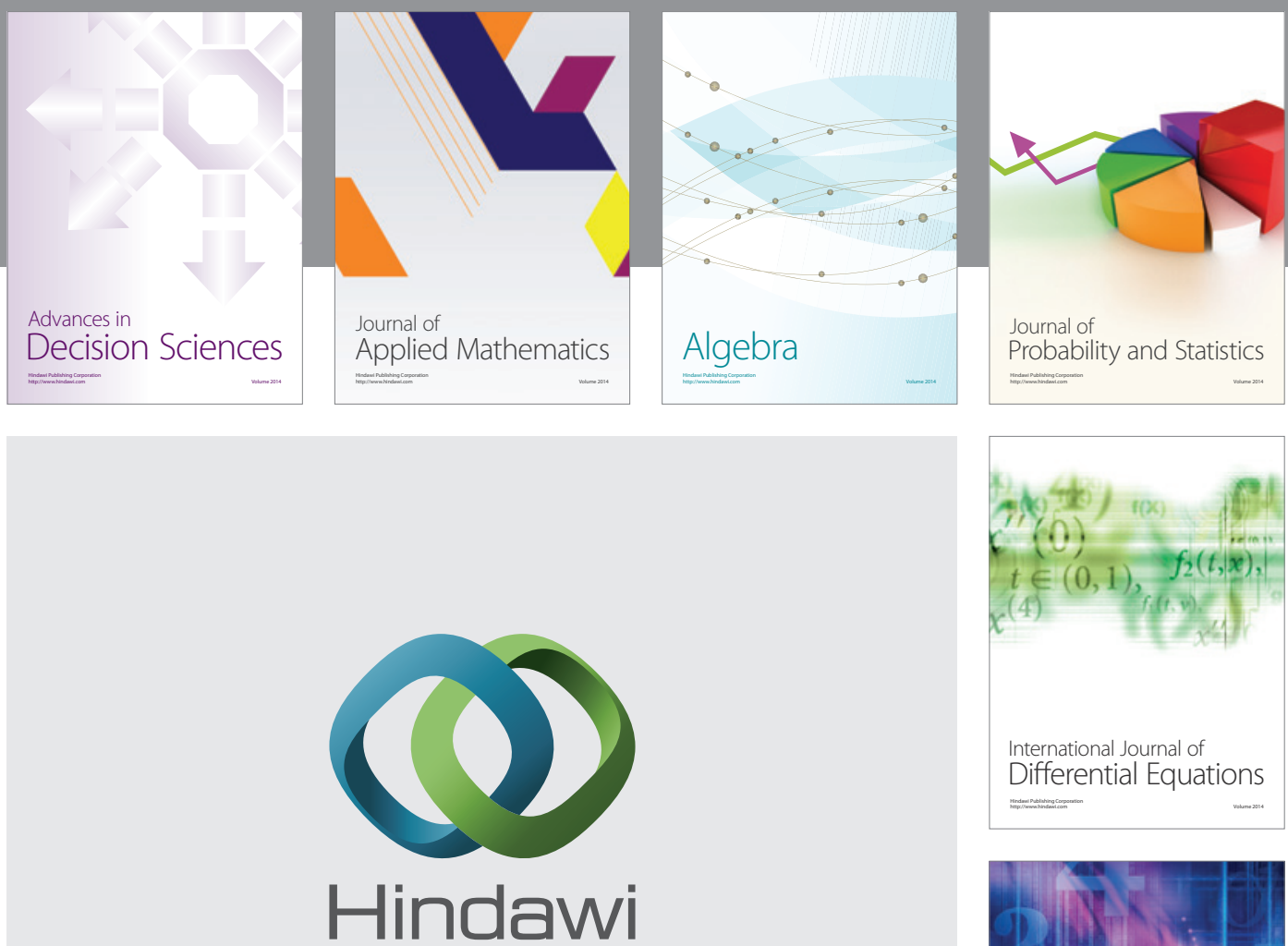

Submit your manuscripts at http://www.hindawi.com
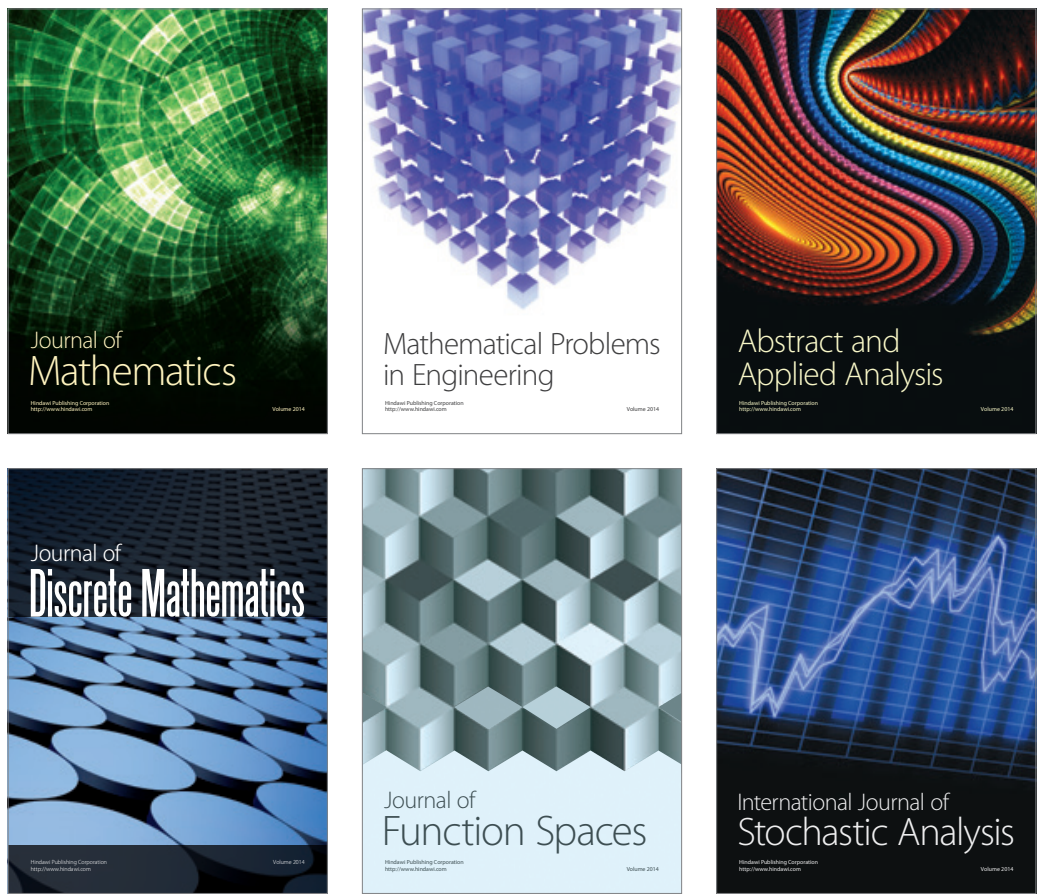

Journal of

Function Spaces

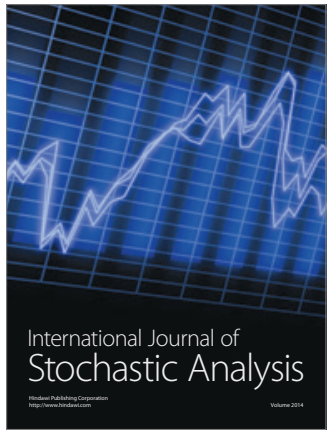

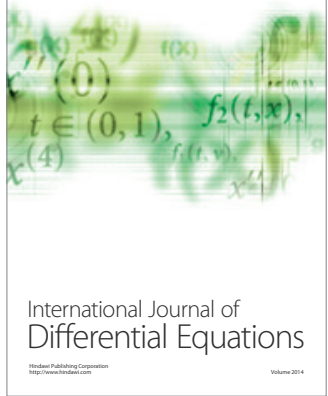
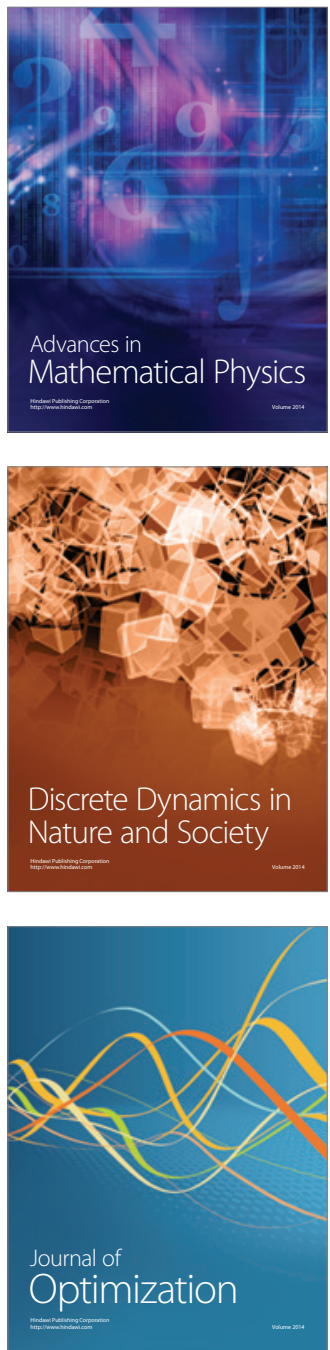